\title{
High average power, diode pumped petawatt laser systems: a new generation of lasers enabling precision science and commercial applications
}

C. Haefner, A. Bayramian, S. Betts, R. Bopp, S. Buck, et al.

C. L. Haefner, A. Bayramian, S. Betts, R. Bopp, S. Buck, J. Cupal, M. Drouin, A. Erlandson, J. Horáček, J. Horner, J. Jarboe, K. Kasl, D. Kim, E. Koh, L. Koubíková, W. Maranville, C. Marshall, D. Mason, J. Menapace, P. Miller, P. Mazurek, A. Naylon, J. Novák, D. Peceli, P. Rosso, K. Schaffers, E. Sistrunk, D. Smith, T. Spinka, J. Stanley, R. Steele, C. Stolz, T. Suratwala, S. Telford, J. Thoma, D. VanBlarcom, J. Weiss, P. Wegner, "High average power, diode pumped petawatt laser systems: a new generation of lasers enabling precision science and commercial applications," Proc. SPIE 10241, Research Using Extreme Light: Entering New Frontiers with Petawatt-Class Lasers III, 1024102 (26 June 2017); doi: 10.1117/12.2281050 


\title{
High average power, diode pumped Petawatt laser systems: a new generation of lasers enabling precision science and commercial applications
}

\author{
C. L. Haefner, A. Bayramian, S. Betts, R. Bopp, S. Buck*, J. Cupal*, M. Drouin*, A. Erlandson, J. Horacek*, J. Horner, J. \\ Jarboe, K. Kasl*, D. Kim, E. Koh, L. Koubikova*, W. Maranville, C. Marshall, D. Mason, J. Menapace, P. Miller, \\ P. Mazurek*, A. Naylon*, J. Novak*, D. Peceli*, P. Rosso, K. Schaffers, E. Sistrunk, D. Smith, T. Spinka, J. Stanley, R. Steele, \\ C. Stolz, T. Suratwala, S. Telford, J. Thoma*, D. VanBlarcom, J. Weiss*, P. Wegner \\ Lawrence Livermore National Laboratory, 7000 East Ave., L-492, Livermore, CA 94550 \\ *ELI-Beamlines, Institute of Physics ASCR, v.v.i., 18221 Prague \\ Email: haefner2@llnl.gov
}

Large laser systems that deliver optical pulses with peak powers exceeding one Petawatt (PW) have been constructed at dozens of research facilities worldwide and have fostered research in High-Energy-Density (HED) Science, High-Field and nonlinear physics [1]. Furthermore, the high intensities exceeding $10^{18} \mathrm{~W} / \mathrm{cm}^{2}$ allow for efficiently driving secondary sources that inherit some of the properties of the laser pulse, e.g. pulse duration, spatial and/or divergence characteristics. In the intervening decades since that first PW laser, single-shot proof-of-principle experiments have been successful in demonstrating new high-intensity laser-matter interactions and subsequent secondary particle and photon sources. These secondary sources include generation and acceleration of charged-particle (electron, proton, ion) and neutron beams, and x-ray and gamma-ray sources, generation of radioisotopes for positron emission tomography (PET), targeted cancer therapy, medical imaging, and the transmutation of radioactive waste $[2,3]$. Each of these promising applications requires lasers with peak power of hundreds of terawatt (TW) to petawatt (PW) and with average power of tens to hundreds of $\mathrm{kW}$ to achieve the required secondary source flux.

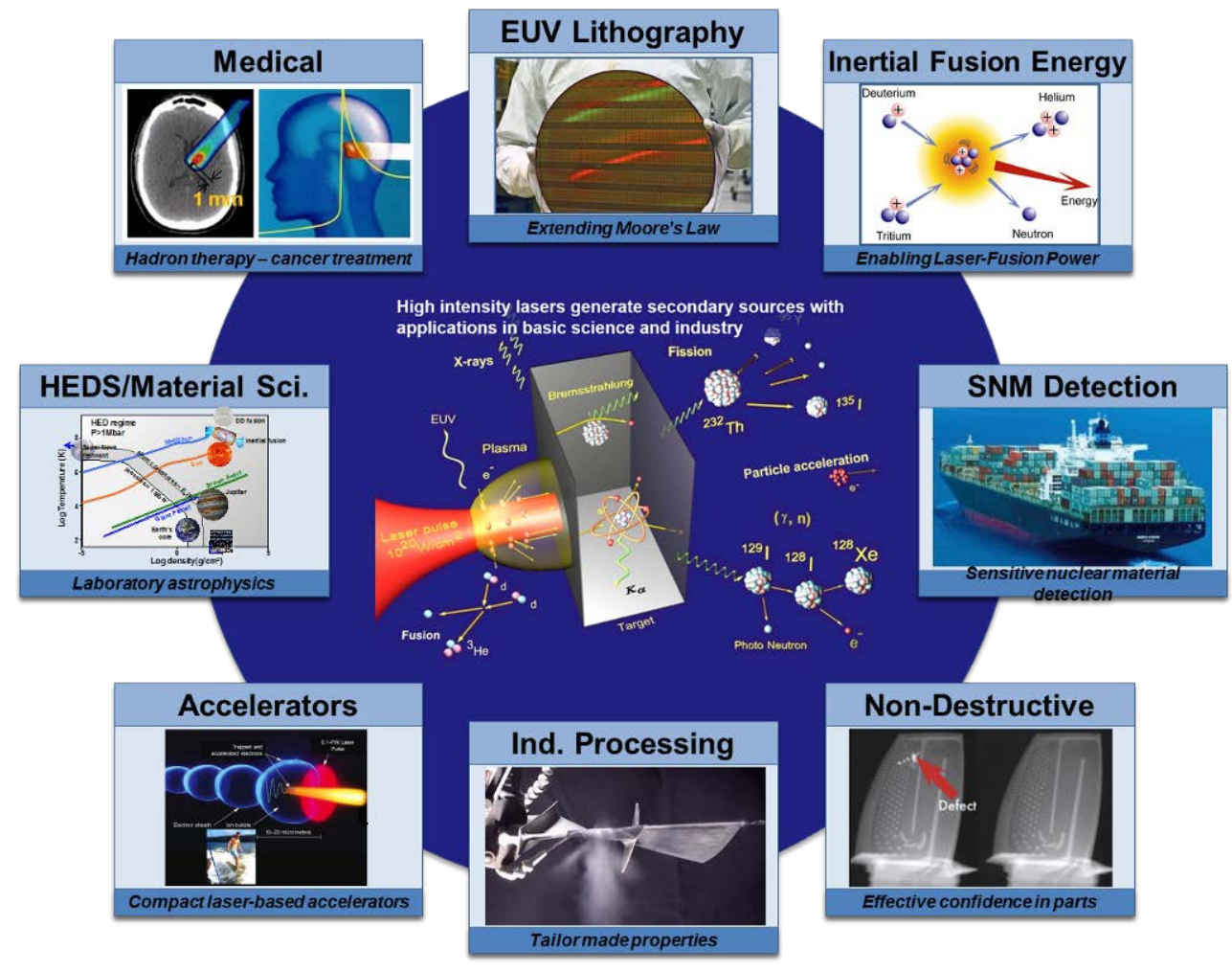

Figure 1: Laser driven secondary sources provide fertile ground for scientific study and commercial applications

Research Using Extreme Light: Entering New Frontiers with Petawatt-Class Lasers III, edited by Georg Korn, Luis O. Silva, Proc. of SPIE Vol. 10241, 1024102 - ( 2017 SPIE

CCC code: $0277-786$ X/17/\$18 - doi: $10.1117 / 12.2281050$ 
Proof of principle experiments underline these applications but have been carried out only at single shot repetition rate for two reasons: first thermal cool-down and thermal recovery of the laser driver and second, manual exchange of the target. In fact, many of today's user facilities rely on flash lamp pumped petawatt laser technology, and have therefore been constrained to push the repetition rate and its associated flux of the secondary source to levels relevant for commercial applications. Another aspect that requires an increase in repetition rate are experiments that rely on statistics, i.e. physical phenomena that are difficult to measure and have a low signal to noise ratio. Achieving highest intensities, controlling pulse shape in space and time, correcting for drift, and controlling the carrier envelope phase for shortest pulses, require feed-back and closed-loop control.

Recently a new generation of lasers with innovative technologies for thermal management, new optical materials and pulse compressor gratings and advanced computer controls have been developed to enter the new regimes. Current pulsed high energy laser systems typically deliver $<10 \mathrm{MJ} /$ day, with two high energy laser systems pushing beyond that barrier: The high energy DiPOLE100 laser system designed and developed for the Czech HiLASE center by the UK's Science and Technology Facilities Council, Rutherford Appleton Laboratory, relies on amplification in cyro-cooled Yb:YAG and recently demonstrated delivery of nanosecond laser pulses at $1 \mathrm{~kW}$ of average power and $10 \mathrm{~Hz}$ repetition rate; and the High-repetition-rate Advanced Petawatt Laser System (HAPLS) at the Lawrence Livermore National Laboratory (LLNL) recently delivered to the European Extreme Light Infrastructure (ELI) Project in Czechia which demonstrated continuous delivery of chirped laser pulses with energy exceeding 16J at 3.3 $\mathrm{Hz}$, compressed pulse duration of 28fs which is equivalent to a peak power of $\sim 0.5$ PetaWatt/pulse. Both DiPOLE and HAPLS rely on the face-gas-cooling of amplifier slabs that was pioneered at LLNL in 1983 and reduced to practice on the Mercury laser system. Mercury delivered $65 \mathrm{~J}$ at $10 \mathrm{~Hz}$ with $10 \mathrm{~ns}$ pulse duration. Mercury and HAPLS use helium (He) gas that is held at room temperature; DiPOLE100 uses cryogenic He-gas $(\sim 150 \mathrm{~K})$ to reduce the saturation florescence to acceptable levels.

HAPLS is a fully integrated petawatt class laser system. Once installed and integrated with the ELI facility, and ramped to its final design performance of 30J, 30fs, $10 \mathrm{~Hz}$, HAPLS will be the world's highest average power Petawatt laser system, delivering 300Watts of Petawatt peak pulses, and $\sim 1.1 \mathrm{MJ} /$ hour.

The HAPLS (see Figure 1) is composed of two major subsystems: a short pulse, broadband, chirped pulse amplification beamline and an advanced high-energy diode-pumped solid-state laser system (pump laser beamline - PLBL) that are both controlled by a sophisticated control system. The pump laser energizes the final power amplifier of the short pulse beamline (SPBL). The PLBL is designed to produce laser pulses at wavelength $1053 \mathrm{~nm}$ with up to $200 \mathrm{~J}$ and repetition rate $10 \mathrm{~Hz}$. Laser pulses are subsequently frequency doubled and delivered to the high energy Ti:sapphire amplifier which is designed to produce a minimum of $45 \mathrm{~J}$ per pulse at the input to the final pulse compressor.

The pump laser consists of a front end, a preamplifier and the final power amplifier. The design of the pump laser front end incorporates technologies demonstrated on NIF and the Mercury laser system and are described elsewhere [6]. Pulses are temporally shaped to produce a 20 ns square pulse at the pump laser system output. The preamplifier consists of a diode pumped, linear regenerative amplifiers, followed by a diode pumped, ring regenerative amplifier delivering an energy up to $1 \mathrm{~J}$ per pulse. Pulses are then formatted from round to rectangular, and injected into the power amplifier. The power amplifier consists of two amplifier heads that are pumped by four High-Power Intelligent Laser Diode System jointly developed by LLNL and Lasertel Inc [7]. It provides $\sim 800 \mathrm{~kW}$ peak power per diode array in a 300 us pulse width at repetition rate up to $20 \mathrm{~Hz}$ and in a $5.6 \times 13.8 \mathrm{~cm}^{2}$ beam area. HILADS is the highest peak power and brightest pulsed diode light delivery system in the world. 
Equally critical to high average power operation is the removal of heat from the amplifier head. Efficient heat management of both the HAPLS pump laser and main Ti:Sapphire amplifier is enabled by a multi-slab amplifier design cooled by room temperature, high flow speed helium gas [6, 7]. Turbulent flow efficiently extracts heat from the extraction surfaces of the laser head, producing a longitudinal thermal gradient to minimize thermally induced wavefront distortion. A solid-state edge cladding is used to minimize parasitic amplified spontaneous emission in both the SPBL and the PLBL. The pump laser amplifiers are gas-cooled neodymium doped APG-1 laser glass slabs similar to previous LLNL designs [6]. The two gas-cooled amplifiers are in a 4-pass, angularly-multiplexed, passive-polarization-switched architecture that is image relayed to minimize modulation of the beam and optimize efficiency [8]. Finally, the PLBL output is relay imaged to the frequency-doubler that uses low absorption, large aperture lithium triborate (LBO) frequency converters. At average power, a conversion efficiency of $>75 \%$ was achieved using no deformable mirror in the PLBL. Higher conversion efficiencies can be achieved by integrating an adaptive mirror in the pump laser cavity and further correcting the PLBL wavefront.

The short pulse beamline relies on broadband chirped pulse amplification (CPA) in Titanium doped Sapphire (Ti:Sa). The front end consists of a dual-CPA, commercial short pulse laser system operating at $100 \mathrm{~Hz}$. Temporal contrast enhancement is achieved through an integrated cross-polarized wave generation stage that delivers between $90 \mathrm{~dB}$ and $110 \mathrm{~dB}$ temporal contrast dependent on configuration. The pulse is then stretched to 1ns in an Offner-triplet stretcher and further amplified in a diode pumped booster amplifier, also operating at $100 \mathrm{~Hz}$. Following the front end, the short pulse beam is amplified in an 8-pass preamplifier pumped by a commercial diode-pumped solid-state laser. Denoted the Alpha amplifier, it produces pulses with energy $\sim 0.5 \mathrm{~J}$ at $10 \mathrm{~Hz}$. Amplification to the full HAPLS output energy takes place in the Beta amplifier, a four-pass cavity configuration containing an amplifier head with several gas-cooled Ti:Sa slabs. Both LLNL-designed amplifiers are fully relay-imaged to preserve beam quality, lower B-integral, and increase efficiency.

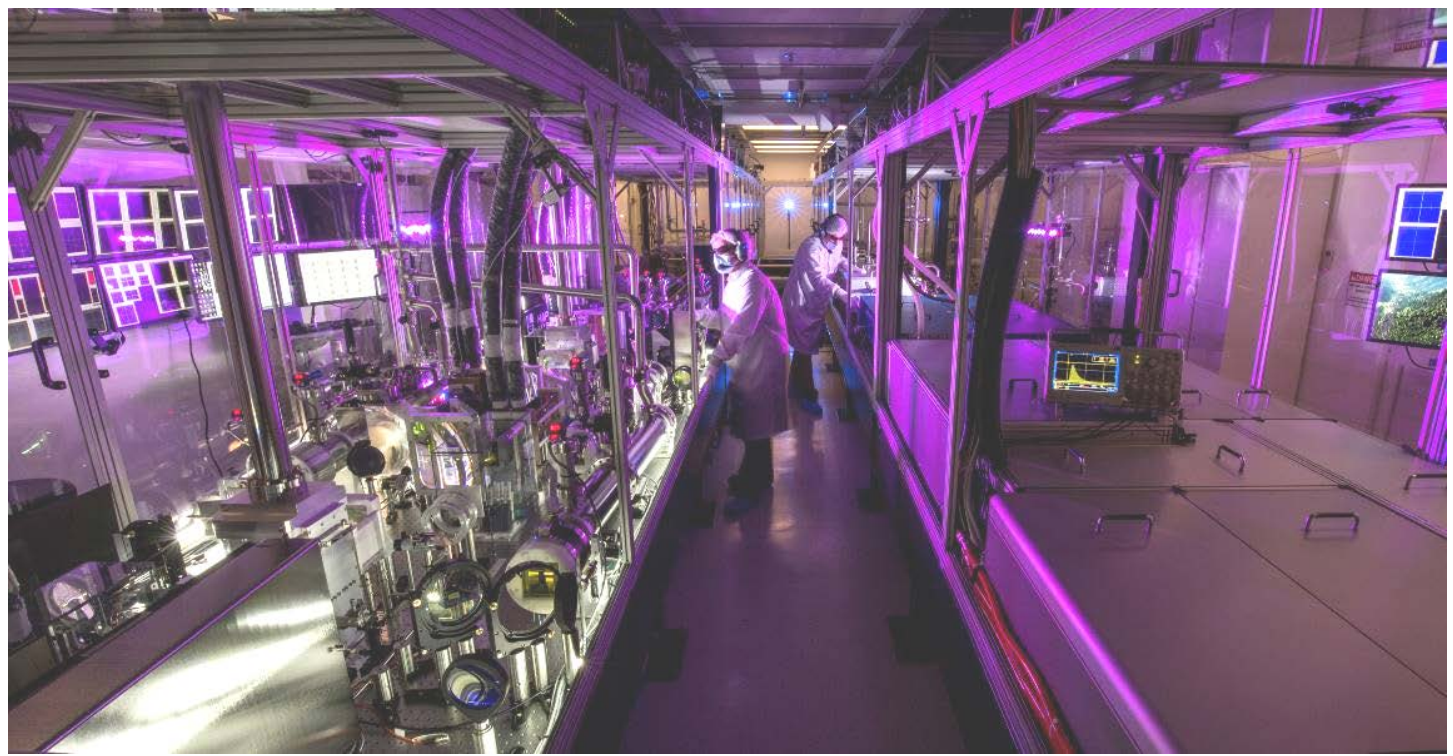

Figure 2: The HAPLS laser laboratory showing the compact and modular approach to this high efficiency high average power laser system

These laser systems were commissioned utilizing an automated control system which managed personnel and machine safety at full repetition rate while displaying and archiving all pertinent data for future users of the output and for monitoring the laser health. Performance diagnostics at the beta amplifier laser output deliver benchmark data for the short pulse system. Using the full-aperture of the main beam, a diagnostic beam is sampled and down-collimated into a small-aperture compressor. Its 
output is split into channels for various pulse diagnostics, including SPIDER [8] to measure pulse duration, cross-correlator to measure temporal contrast, and nodes for energy, spectrum, beam nearfields and far fields to fully characterize each shot.

HAPLS is being ramped in performance in a risk balanced approach. In multiple phases, the output energy is ramped in a single shot configuration, then the average power is increased and laser performance assessed and benchmarked against the laser performance models. Then energy is further ramped, then average power, and so forth. Example data from one of the commissioning runs is shown in Figure 3 left, where the average energy was $16 \mathrm{~J}$ with an rms stability of $2.4 \%$. SPIDER data taken simultaneously is shown in the inset, demonstrating an average FWHM pulse duration of 28.6fs with an rms deviation of 1.4fs. The results shown in Figure 3 right are raw pulse retrieval data for 12000 shots without filtering or averaging. The temporal FWHM from the SPIDER reconstruction for each shot is plotted versus time to indicate the level of compressed-pulse stability achieved. The mean pulse duration is consistent with the measured spectral bandwidth and is $\sim 1.2$ times the transform limit. Shorter duration pulses are possible, and with an output bandwidth of 88nm demonstrated.
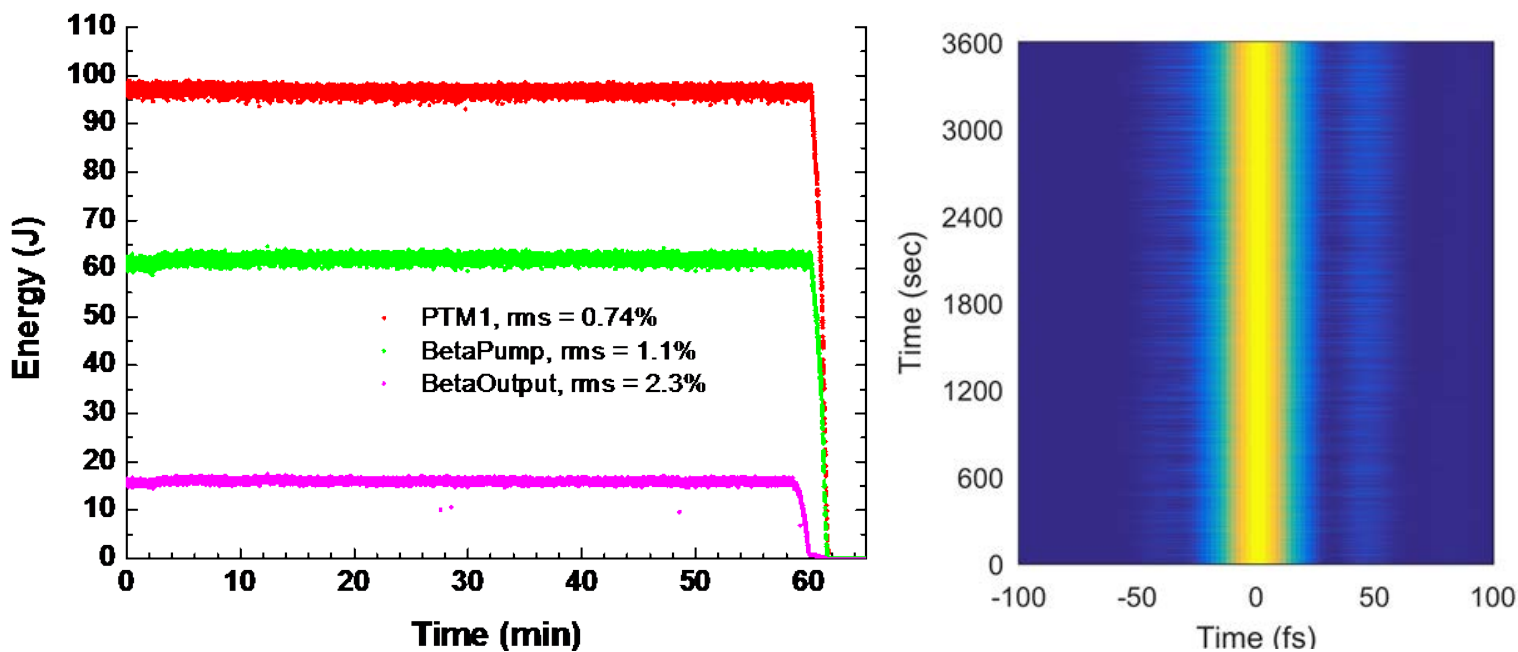

Figure 3: left: Raw data from one of the $1 \mathrm{hr}$ commissioning runs of the HAPLS laser system showing the energy of the pump laser (PTM1), the frequency converted light delivered to the short pulse power amplifier (BetaPump) and the output of the short pulse amplifer output (BetaOutput); right: FWHM pulse duration (28.6fs, reconstructed via SPIDER) stability at $3^{1 / 3} \mathrm{~Hz}$.

Full system laser energy performance models facilitate understanding of system dynamics and allows forecasting final optimized performance. Thermal models allow for optimization of heat deposition and thermal management throughout the laser system, including thermally-induced wavefront effects.

The experimental results confirmed the laser performance models and demonstrated fully integrated operation. HAPLS will be installed at the ELI-Beamlines facility including integration with the full scale, vacuum high average power compressor using advanced LLNL grating technology [9].

HAPLS is designed to provide a flexible experimental platform to ELI users for extended data collection sessions that enable today's proof-of-principle experiments to be pushed towards high fidelity data acquisition, including reduction to practice of commercial secondary source applications (see Figure 4). HAPLS is the first system that crosses the $10 \mathrm{MJ} /$ day threshold that sets applications from today's state-of-the-art laser systems apart. 


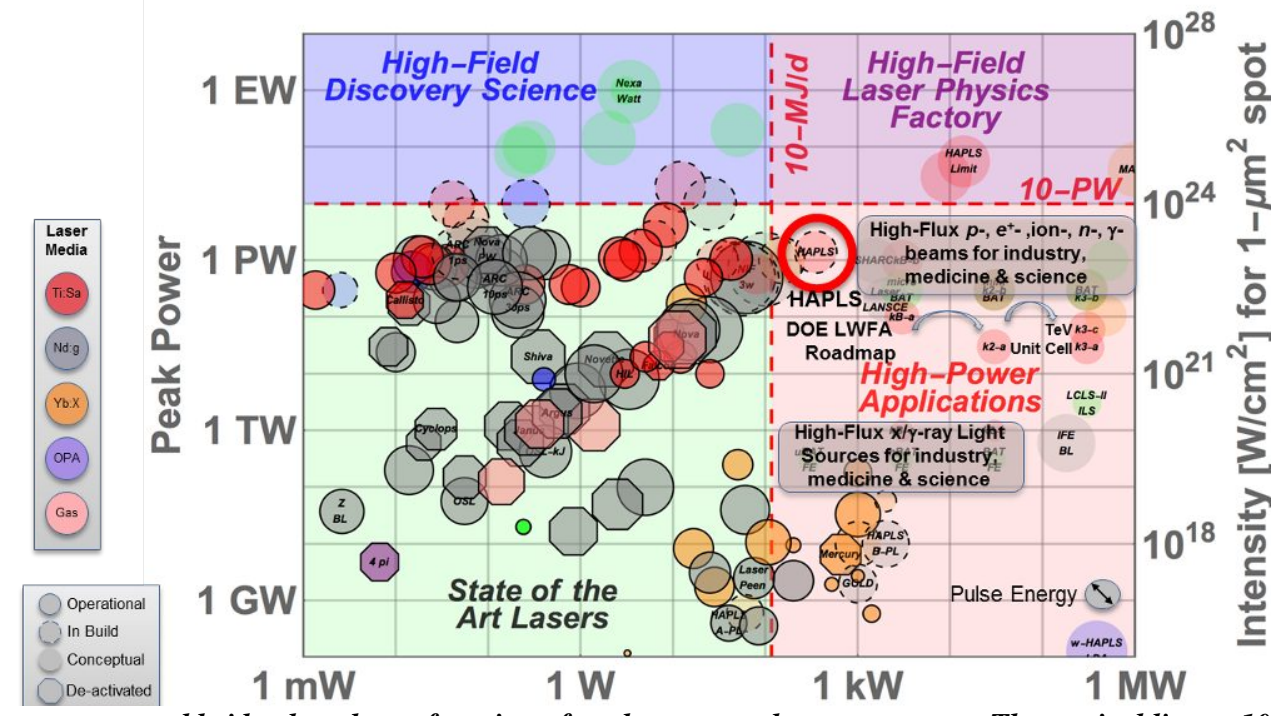

Figure 4: Laser systems worldwide plotted as a function of peak power and average power. The vertical line at 10 $M J /$ day sets lasers that support proof-of-principle experiments apart from the driver needs for applications. The 10PW peak power line is set at around the limit that today's grating technology (meter scale) defines.

This work was performed under the auspices of the U.S. Department of Energy by Lawrence Livermore National Laboratory under Contract DE-AC52-07NA27344, Lawrence Livermore National Security, LLC

\section{References}

1. M. D. Perry, et al., "Petawatt laser pulses," Opt. Lett. 24, pg. 160-162 (1999).

2. G. A. Mourou, G. Korn, W. Sandner, J. L. Collier, "Extreme Light Infrastructure-Whitebook, Science and Technology with Ultra-Intense Lasers”, http://old.eli-beams.eu/wp-content/uploads/2011/10/ELI-Book_neues_Logo-edited-web.pdf

3. Y. Izawa, et al., "High Power Lasers and Their New Applications,” J. Opt. Soc. Korea 12, p. 178-185 (2008).

4. A. Bayramian, et al., "High Energy, High Average Power, DPSSL System For Next Generation Petawatt laser Systems,” CLEO, 2016, OSA.

5. E. Sistrunk, et al., “All Diode-Pumped, High-repetition-rate Advanced Petawatt Laser System (HAPLS),” CLEO, 2017, OSA.

6. A. Bayramian, et al., "The Mercury project: A high average power, gas-cooled laser for inertial fusion energy development,” Fusion Sci. Tech. 52, 383-387 (2007).

7. $\quad$ E. Fulkerson, et al., “Pulsed power system for the HAPLS Diode Pumped Laser System,” Pulsed Power Conference (PPC), 2015 IEEE, pp. 1-6. 2015 R\&D100 Award for "High-Power Intelligent Laser Diode System (HILADS) http://www.rdmag.com/award-winners/2015/10/intelligent-laser?cmpid=horizontalcontent

8. A. Bayramian et al., "Compact, efficient laser systems required for laser inertial fusion energy," Fusion Sci. Tech. 60, 28-48 (2011).

9. C. Iaconis and I. A. Walmsley, "Self-Referencing Spectral Interferometry for measuring Ultrashort Optical Pulses,” IEEE J. Quant. Electron. 35, p. 501-509 (1999).

10. D. A. Alessi, et al., “Active cooling of pulse compression diffraction gratings for high energy, high average power ultrafast lasers,” Optics Express (accepted) 2016. 